Supporting Information 


\title{
Enhanced Interaction of Optical Phonons in h-BN with Plasmonic Lattice and Cavity Modes
}

\author{
Xianglian Song ${ }^{l, 2}$, Sina Abedini Dereshgi ${ }^{1}$,Edgar Palacios ${ }^{1}$, Yuanjiang Xiang, ${ }^{2,3, *}$, Koray Aydin $^{l, *}$ \\ 1 Department of Electrical and Computer Engineering, Northwestern University, Evanston, IL 60208, United States \\ 2 International Collaborative Laboratory of 2D Materials for Optoelectronic Science \& Technology of Ministry of Education, \\ Institute of Microscale Optoelectronics (IMO), Shenzhen University, Shenzhen 518060, China \\ 3 Key Laboratory for Micro/Nano Optoelectronic Devices of Ministry of Education and Hunan Provincial Key Laboratory of \\ Low-Dimensional Structural Physics and Devices, School of Physics and Electronics, Hunan University, Changsha, 410082, \\ China
}

* Corresponding authors

* Dr. Koray Aydin. E-mail: aydin@northwestern.edu

* Dr. Yuanjiang Xiang. E-mail: xiang78@hnu.edu.cn 


\section{TABLE OF CONTENTS}

\section{A. Absorption of pure Ge layer}

Figure S1. Absorption of Ge layer on background metal only.

\section{B. Dispersion of the structure excluding hBN}

Figure S2. FDTD simulated absorpion contour maps of the plasmonic cavity without $h B N$ at varied parameters. Figure S3. Total electric field magnitude at the cross-section of the design without $h B N$ at varied modes.

C. Tunable resonance of the structure

Figure S4 Fitting the measured resonant peaks to the absorption contours for extra Ge thickness and periodicity.

\section{Oscillator model and strong coupling}

Figure S5 Fitting the oscillator model to the resonances from modes coupling in Figure 3 of the main manuscript.

\section{E. $P_{a b s}$ distribution of each layer}

Figure S6 Contribution of each layer in absorption $\left(P_{a b s}\right)$.

Figure $S 7$ Contribution of $P_{\text {abs }}$ from in-plane and out-of-plane phonon modes to the structures with varied Ge thicknesses.

F. SEM surface morphology of fabricated samples

Figure S8 Selected surface morphologies of Au gratings deposited on hBN thin film for varied width ratios. Figure S9 Surface morphologies of Au gratings deposited on hBN thin film for varied periodicity.

\section{G. Oblique incidence sensitivity}

Figure 10 Effect of incidence to the device 


\section{A. Absorption of pure Ge layer}

Here, three kinds of Ge thin films with various thicknesses $\left(t_{G e}\right)$ were fabricated through e-beam evaporation, on which $\mathrm{hBN}$ thin films were transferred and Au gratings were fabricated on top subsequently. The absorption of Ge layer on background metal (150 nm Au) is shown in Figure S1. The thickness of Ge is calculated through matching measured absorption peaks with simulated ones, which is further verified by SEM cross section images. The real and imaginary parts of the permittivity of Ge is set constant as 4.007 and 0 respectively during FDTD process. As inferred from Figure $S 1$ and Figure 2, the resonant wavelength can be tuned by the real part of permittivity and thickness of Ge layer, while the bandwidth and intensity are mainly determined by imaginary part of permittivity. Besides, with Au gratings on the top, the peak originated from Fabry-Perot resonance is enhanced, especially for Ge layer with thicknesses of 470 and $650 \mathrm{~nm}$. It should be noted that the peak intensity is related to defects and pinholes in Ge films deposited through e-beam evaporation process. The small discrepancy is attributed to loss coefficients introduced to Ge from deposition processes and hBN roughness.

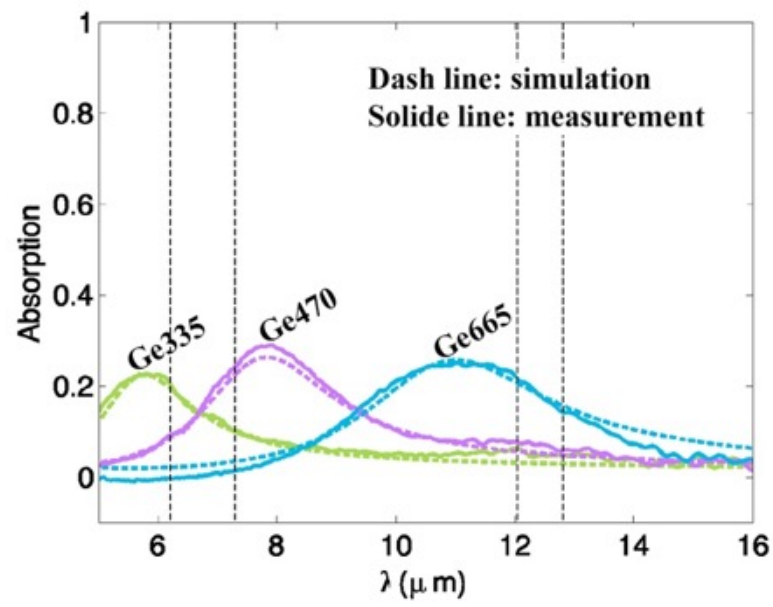

Figure S1 Absorption of Ge layer on background metal only

\section{B. Dispersion of the structure excluding hBN}

The dispersion contour counterparts of Figures $2 c, 3 a$ and $3 d$, without hBN layer, are provided here. The response of this plasmonic cavity to the changes in Ge thickness, filling factor and periodicity of the Au gratings are respectively illustrated in Figures $S 2 a-c$.

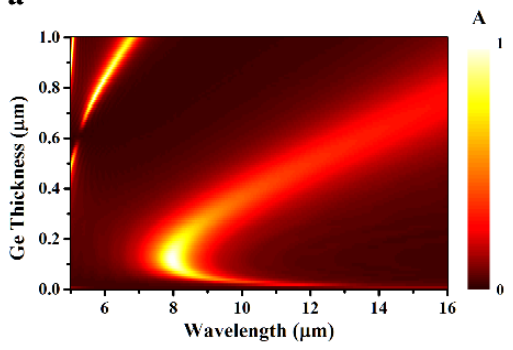

b

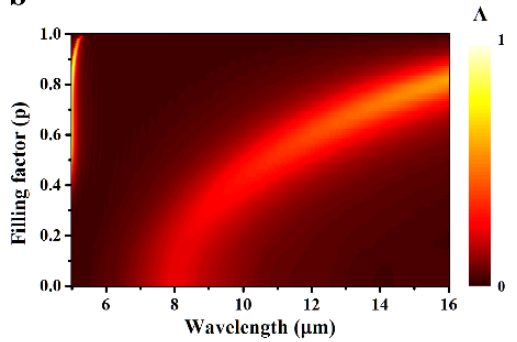

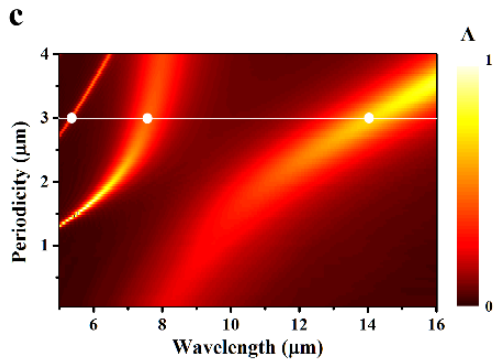

Figure S2 FDTD simulated absorpion contour maps of the plasmonic cavity without hBN as a function of (a) Ge thickness in the cross-section of the plasmonic cavity without $h B N(b)$ filling factor $(p=w / \Lambda)$, with a constant periodicity $(\Lambda \approx 1.3 \mu \mathrm{m})$ and varied grating width $\boldsymbol{w}$ and $t_{G e}=470 \mathrm{~nm}$, and $(c)$ periodicity $\Lambda(\mu \mathrm{m})$, with constant filling factor of Au gratings $(p \approx 0.4)$ and $t_{G e}=470 \mathrm{~nm}$. The marked points are used for mode analysis in Figure S4. 
Figure $S 2 a$ suggests that several plasmonic modes can be excited by varying Ge thickness, the fundamental and second order cavity plasmon modes in particular. Given that $t_{G e}$-dependent simulations are carried out for the purpose of choosing Ge thickness and the main scope of this study is the effect of grating on $\mathrm{hBN}$, no further discussion is pursued for the observed modes. For the result of Figure $S 3 b$, we observe the fundamental and higher order cavity mode being excited in the cavity. For Figure $S 3 c$, cavity and lattice modes are accessible. The three dots marked in this panel are used for the field profile results in Figure $S 4$ below, where $\Lambda \approx 1.3 \mu \mathrm{m}$ is chosen to encompass all of the three different modes at different resonance wavelength values.

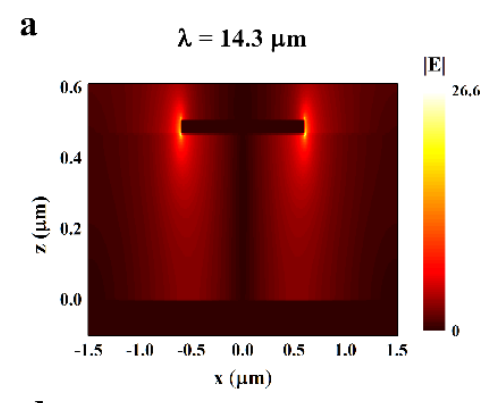

d

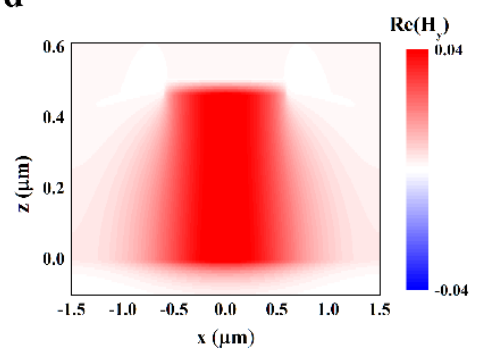

b

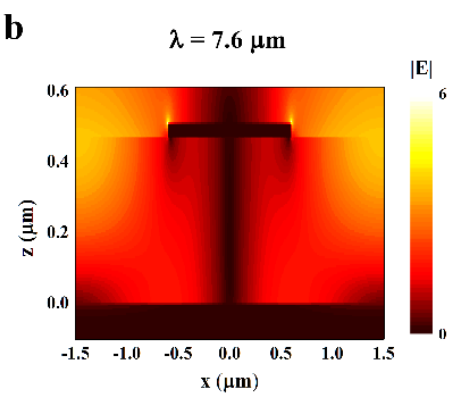

e

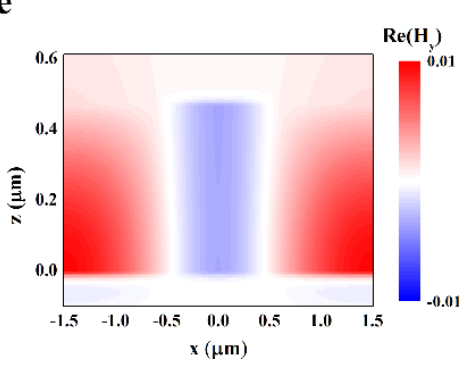

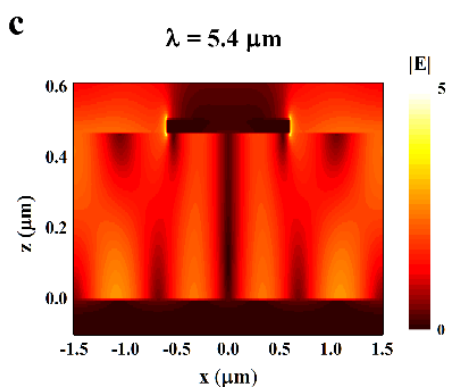

f

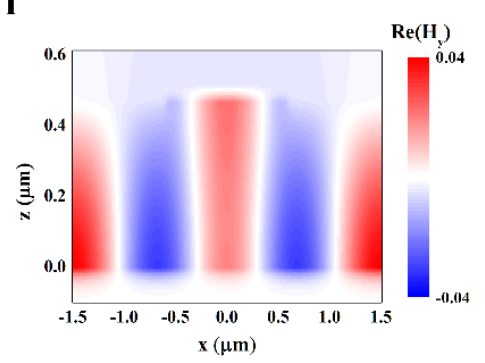

Figure S3 Total electric field magnitude at the cross-section of the design without $h B N$ at (a) fundamental cavity mode $\lambda=$ $14.3 \mu \mathrm{m}$, (b) fundamental lattice mode $\lambda=7.6 \mu \mathrm{m}$ and (c) higher order cavity mode $\lambda=5.4 \mu \mathrm{m}$. (d)-(f) Real part of the magetic field in the $x z$ device cross-section corresponding to panels (a) to (c). The design parameters are illustrated in Figure S2c.

\section{Tunable resonance of the structure}

The multiple resonances obtained from absorption spectra are ascribed to phonon polaritons enhanced in Fabry-Perot cavity. These morphologies of resonant peaks are dominated by Ge thickness $\left(t_{G e}\right)$, unit periodicity $(\Lambda)$, and grating ratio $(p)$. As presented in Figure $S 4(\mathrm{a})$, resonant marks are extracted from FTIR absorption spectrum for grating ratio $(p)$ varied from $0 \sim 1$, with $t_{G e}=650 \mathrm{~nm}$ and $\Lambda=1.3 \mu \mathrm{m}$, which are aligned well to FDTD contour. However, the anti-splitting resonance from out-of-plane phonon polaritons enhancement are merged together as that for $t_{G e}=650 \mathrm{~nm}$, as well as the weak peaks originating from minor optical phonons. The is attributed to the mode broadening due to fabrication non-idealities. While keeping $t_{G e}=470 \mathrm{~nm}, \Lambda=1.8 \mu \mathrm{m}$, the enhancement of minor OPhs is quite strong for low filling factor values, which merge into resonant peaks approaching upper $\lambda_{L O}$. In fact, the coupling of in-plane phonon polaritons switches from approaching upper $\lambda_{T O}$ to $\lambda_{L O}$, and similarly, the coupling of out-of-plane phonon polaritons are enhanced, switching from approaching lower $\lambda_{T O}$ to $\lambda_{L O}$, as the clear anti-cross resonance manifests. 

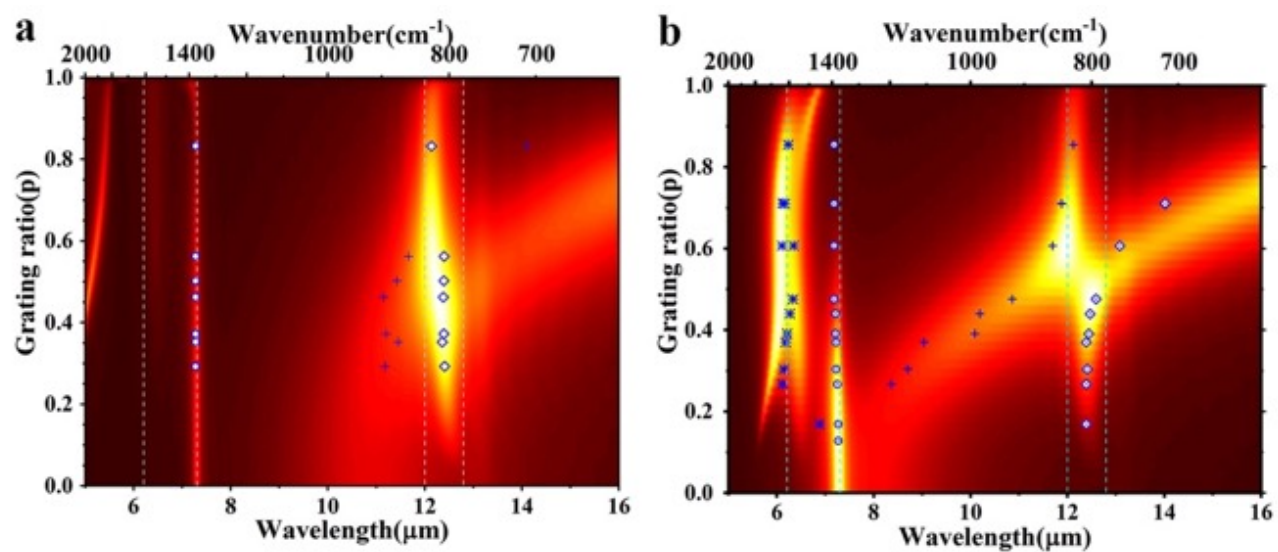

Figure S4(a) Thickness of Ge is kept at $650 \mathrm{~nm}$, periodicity $\Lambda=1.3 \mu \mathrm{m}$, where filling factor $p$ is swept from 0 to 1 . (b) Thickness of Ge is kept at $470 \mathrm{~nm}$ and periodicity $\Lambda=1.80 \mu \mathrm{m}$, filling factor $p$ is swept from 0 to 1 . All the markers are extracted from resonant peaks measured in FTIR.

\section{Oscillator model and strong coupling}

The oscillator model of the plasmonic and 1-Phz modes are illustrated in Figure $S 5 a$ where these two modes are independent. Figure $S 5 b$ represents the latter two modes in coupled condition schematically. Figures $5 c$ and $d$ illustrated the fitted oscillator model that tracks the absorption peaks on the contour plots obtained from FDTD simulations.

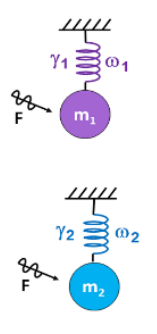

(a)

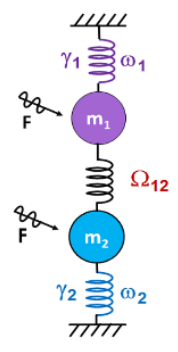

(b)

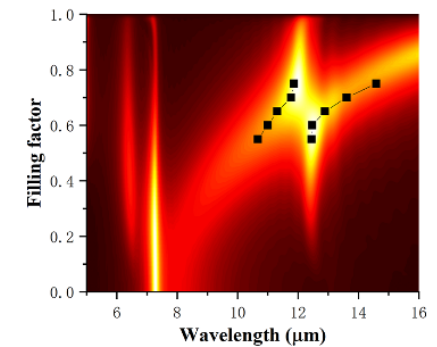

(c)

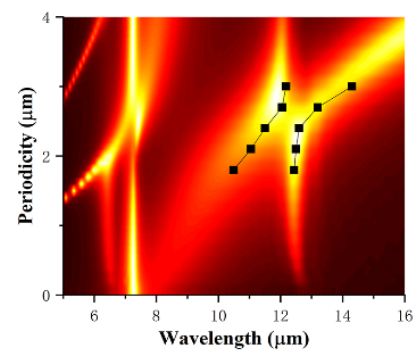

(d)

Figure S5 The oscillator model of independent plasmonic and 1-Phz modes (a), and the two modes couplling (b). (c) \& (d) Fitting oscillator model to the resoances from coupled plasmonic and 1-Phz modes, correspsonding to Figure 3 a and

Figure $3 d$ in the main manuscript respectively.

The coupling strength is a critical parameter here to describe the enhanced interaction of optical phonons and plasmonic modes in our system, further discussion is put forth in the following. The coupling strength $(g)$ can be calculated through the following equation: 


$$
g=\frac{\hbar \Omega}{2}
$$

where $\hbar \Omega$ refers to Rabi splitting energy, which could be obtained from the absorption contour, around the coupling peaks. For strong coupling, the system should satisfy the following criteria [1,2]:

$$
\hbar \Omega>\frac{\hbar \gamma_{p l}+\hbar \gamma_{p h}}{2}
$$

Where $\gamma_{p l}$ and $\gamma_{p h}$ are the dissipation rates of the un-coupled plasmonic and phonon modes respectively. Specifically, as we calculated from the oscillated model, $\hbar \gamma_{p l}=130 \mathrm{~cm}^{-1}$ at $\omega_{1}=825.1 \mathrm{~cm}^{-1}$ for the single plasmonic mode and $\hbar \gamma_{p h}=8 \mathrm{~cm}^{-1}$ at $\omega_{2}=876.48 \mathrm{~cm}^{-1}$ for the major optical phonon from the out-of-plane direction (1-Phz). The splitting energy is $\hbar \Omega_{p l-p h}=2 \mathrm{~g}=76 \mathrm{~cm}^{-1}$ at $\omega_{r}=831.5 \mathrm{~cm}^{-1}$, as also seen from the main manuscript.

Thus, the following equation can be obtained,

$$
\frac{\hbar \gamma_{p l}+\hbar \gamma_{p h}}{2}=\frac{130+8}{2}=69<\hbar \Omega_{p l-p h}=76\left(\mathrm{~cm}^{-1}\right)
$$

in which the total loss is smaller than the Rabi splitting energy. We can conclude here that the enhanced absorption we observe here from absorption enhancement of main out-of-plane optical phonons (1-Phz) is strongly coupled to plasmonic lattice mode. This strong coupling is realized thanks to the very sharp optical phonon resonance with small broadening factor.

There are three regimes of exotic phenomena for light-matter interaction. Normalized strong coupling strength $\eta[3]$ is defined as the ratio of light-matter coupling strength $g$ to the mid-gap frequency $\omega_{0}$,

$$
\eta=\frac{g}{\omega_{0}}
$$

Strong coupling is usually referred to the case when $\eta<0.1$, ultrastrong coupling (USC) regime where $0.1<\eta<1$, and deep strong coupling (DSC), $\eta>1[4,5]$. Thus, we can calculate the normalized coupling strength for the splitting region from $1-P h z$,

$$
\eta=\frac{g}{w_{r}}=\frac{76 / 2}{831.1}<0.1
$$

Therefore, only strong coupling takes place for our case since the interaction is not strong enough. As a result, rotating wave approximation (RWA) does not break down and the problem can be treated as a perturbation, neglecting the counter-rotating term (CRT). Detailed analysis of strong coupling is added to the part ' $D$ ' in the Supporting Information as well.

In order to further strengthen the coupling and potentially push it to the USC and DSC regime, one needs to design structures that support sharper resonance peaks to accommodate the sharp resonances of optical phonons. While optical phonons are high-quality-factor resonances, it is a well-established fact that plasmonic resonances, specifically plasmonic lattice resonances, are low-quality-factor resonances and they would not yield USC. Mie resonances possess similar and sometimes lower quality factors to the plasmonic ones [6]. Photonic crystals are also known to demonstrate high quality factors and might be one possible path toward stronger coupling [7]. More recently, there are reports on bound states in continuum (BIC) resonances that are some of the sharpest resonances observed in literature, exceeding $10^{9}[8,9]$. Coupling of BIC and phonon resonances can lead to USC. We also note that when the resonances are sharp, extra care must be taken to bring two sharp resonances to close vicinity. This requirement can make the design process of the cavity challenging since the resonances might drift away easily by fabrication non-idealities. DSC is challenging to achieve and might require active components to minimize the losses within the resonant system. 


\section{E. $P_{a b s}$ distribution of each layer}

The contribution of each layer is illustrated by $P_{a b s}$ calculation displayed in Figure $S 5 . P_{a b s}$ is used to describe the power absorption per unit volume, which is a function of frequency as bellow,

$$
P_{a b s}=\frac{1}{2} \omega \varepsilon_{0} \operatorname{Im}(\varepsilon)|E|^{2}
$$

These multi resonant peaks mostly attribute to energy absorption in $\mathrm{hBN}$ layer, originating from both coupling enhancement of in-plane and out-of-plane OPhs, including both the main and second modes. The absorption peaks located between upper $\lambda_{T O}$ and lower $\lambda_{L O}$ are ascribed to hBN layer, while the energy dissipated in other layers are very small in contrast.

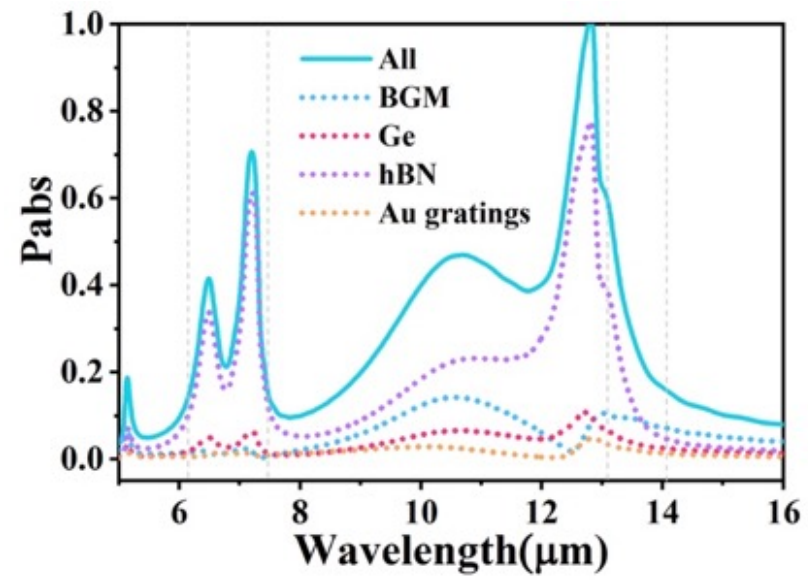

Figure S6 Contribution of each layer in absorption $\left(P_{a b s}\right)$, while the label 'All' corresponds to the whole structure, 'BGM' is short from 'backgroud metal', 'Ge', ' $h B N$ ' and 'Au-grating' correspond to the absorption taking place in Ge, $h B N$ and Au gratings layers respectively.

$P_{a b s}$ is calculated for structures with Au gratings on $\mathrm{hBN}$ at the top of Ge layer with various thicknesses. Multiple sharp resonant peaks originating from in-pane and out-of-plane OPhs are further discussed. The first peak located at $\lambda_{L O, \text { upper }}$ mainly stems from minor out-of-plane 2-Phz OPhs, which decreases while increasing Ge thickness. The second peak located at $\lambda_{\text {TO,upper }}$ is due to the enhancement of in-plane OPhs, whose intensity decrease while Ge thickness increases from 335 to $650 \mathrm{~nm}$. In lower RS band, the coupling of out-of-plane OPhs is enhanced while increasing Ge thickness, and as a result, the intensity of the resonant peak located at $\lambda_{L O, \text { lower }}$ increases. The 2-Phx mode of in-plane OPhs remains almost unchanged with a low intensity for all the three thicknesses.
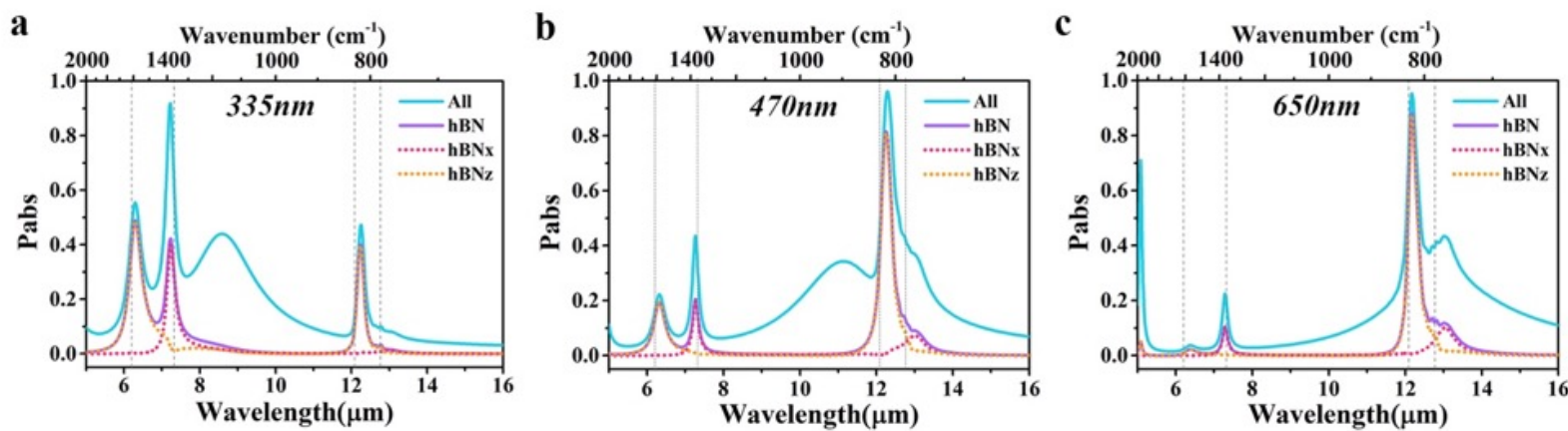

Figure S7 Contribution of Pabs from in-plane and out-of-plane phonon modes to the structures with three different Ge thicknesses, (a) $t_{G e}=335 \mathrm{~nm}$, (b) $t_{G e}=470 \mathrm{~nm}$, (c) $t_{G e}=650 \mathrm{~nm}$. 


\section{F. SEM surface morphology of fabricated samples}

SEM images of the fabricated devices are shown in Figure $S 8$ and $S 9$, acting as supplementary to Figure $3 c$ and $3 f$ respectively in the main manuscript.
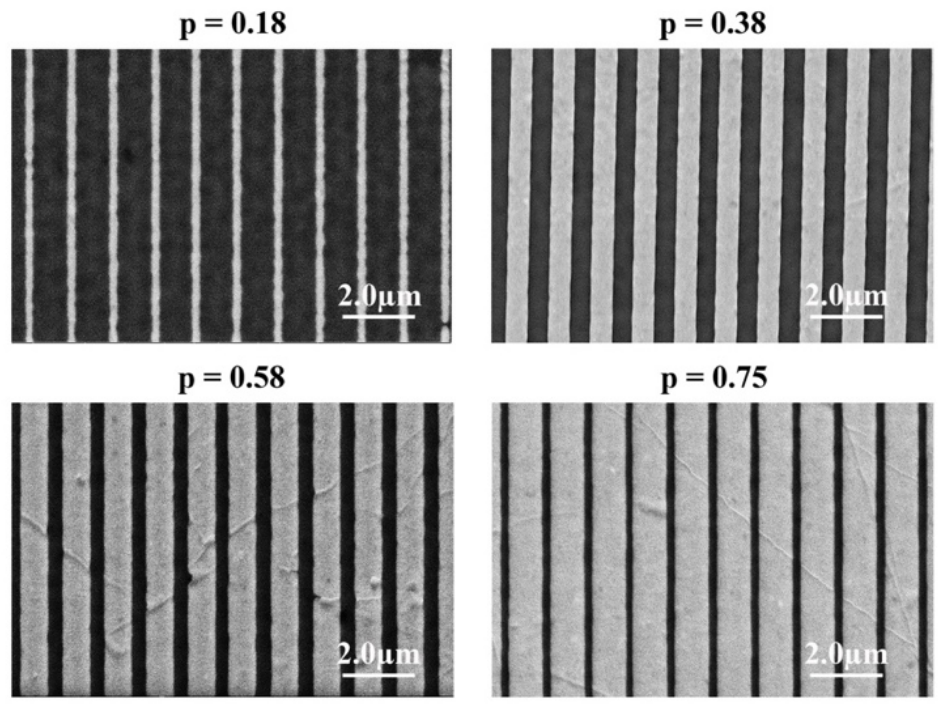

Figure S8 Selected surface morphologies of Au gratings deposited on $h B N$ thin film for width ratio $p=0.18,0.38,0.58$ and 0.75 respectively, where the periodicity ( $\Lambda$ is kept constant at $\Lambda=1.3 \mu \mathrm{m}$, as a supplementary to Figure $3 \mathrm{c}$ in the main manuscript.

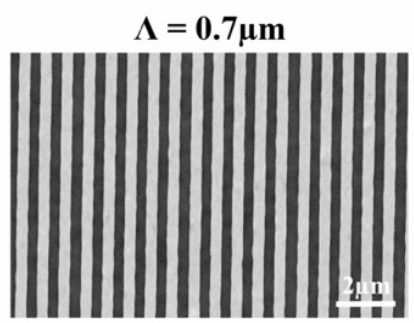

$\Lambda=2.6 \mu \mathrm{m}$

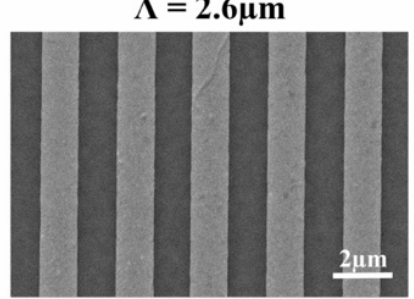

$\Lambda=1.3 \mu \mathrm{m}$

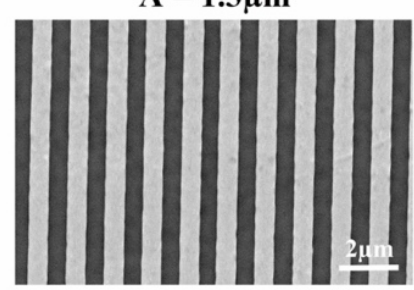

$\Lambda=3.3 \mu \mathrm{m}$
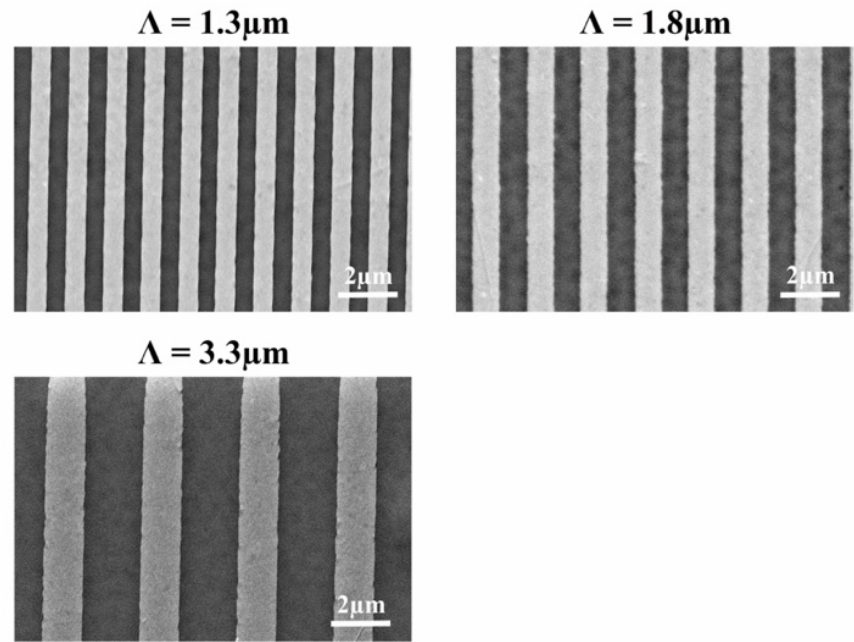

Figure S9 Surface morphologies of Au gratings deposited on $h B N$ thin film for periodicity values $\Lambda=0.7,1.3,1.8,2.6$ and $3.3 \mu \mathrm{m}$ respectively; the width ratio ( $p$ ) is kept constant at $p=0.4 \pm 0.05$, as a supplementary to Figure $3 f$ in the main manuscript.

\section{G. Oblique Incidence sensitivity of the device}

Simulations for various incident angles from $0 \sim 60$ degrees over a broadband wavelength range and the absorption spectra for devices with two Au-grating periodicities $(\Lambda=1.3$ and $2.6 \mu \mathrm{m})$ are shown in Figure S10. At $\Lambda=1.3 \mu \mathrm{m}$, all the present modes are uncoupled in contrast to $\Lambda=2.6 \mu \mathrm{m}$ where we will observe coupling of modes. For normally broadband calculation with a plane wave incidence, the actual incident angle during a simulation changes as a function of wavelength, which poses issues to simulation accuracy and the results would not be reliable. Single wavelength sweeping can be an alternative to solve 
this issue, but it is time consuming. To avoid such inaccuracy and run the simulation within a reasonable time, the broadband Fixed Angle Source Technique (BFAST) source is adopted in the incidence simulation instead.

First, $\Lambda=1.3 \mu \mathrm{m}$ is considered (Figure $S 10 a$ ) to investigate the behavior of the modes independently. Figure S1Oa suggests that the absorption spectrum of the device remains unchanged for incident angles below 15 degrees, since this incident angle is too small to cause significant change in response. When the incident angle is increased from 15 to 60 degrees, the coupling peaks from the main optical phonons in hBN (1-Phx and $1-P h z)$ are not affected dramatically.

The absorption from minor out-of-plane phonon mode (2-Phz), however, is enhanced; absorption intensity increases due to the increase in the intensities of the two modes sandwiching it (i.e., the resonances near $5.5 \mu \mathrm{m}$ and $7.2 \mu \mathrm{m}$ ) as well as the redshift of the mode near $5.5 \mu \mathrm{m}$ that approaches the $2-P h z$ at $6.5 \mu \mathrm{m}$. The minor in-plane phonon modes (2-Phx) are rather stable in frequency and intensity. The peaks near $10 \mu \mathrm{m}$ (plasmonic lattice modes) are negligibly sensitive to incident angle. This mode redshifts slightly when the incident angle increases since the tangential component of momentum increases for higher incident angles. However, due to diffractive nature of the plasmonic lattice that already enhances momentum in all directions, this resonance shift is negligible. Contrarily, the cavity plasmonic modes below $6 \mu \mathrm{m}$ redshift more significantly as the incident angle is increased. This follows from the FP condition for momentum matching that a lower in-plane momentum requires thicker Ge layer to match the resonance condition. This can be understood through the FP momentum matching condition,

$$
\varphi_{\text {Grating }}+2 k_{\|, G e} t_{G e}+\varphi_{A u, b o t t o m}=2 \pi m, m=0,1,2, \ldots
$$

Where $\varphi_{\text {Grating }}$ and $\varphi_{A u, b o t t o m}$ are the reflected phase from the grating and bottom metal and $k_{\|, G e}$ is the tangential component of the wavevector. For higher incident angle $(\theta), k_{\|, G e}=(2 \pi / \lambda) \sin \theta$ increases. Since $t_{G e}$ is the same, the resonance is realized in a longer wavelength $\lambda$ to maintain the resonance value for $2 k_{\|, G e} t_{G e}$.
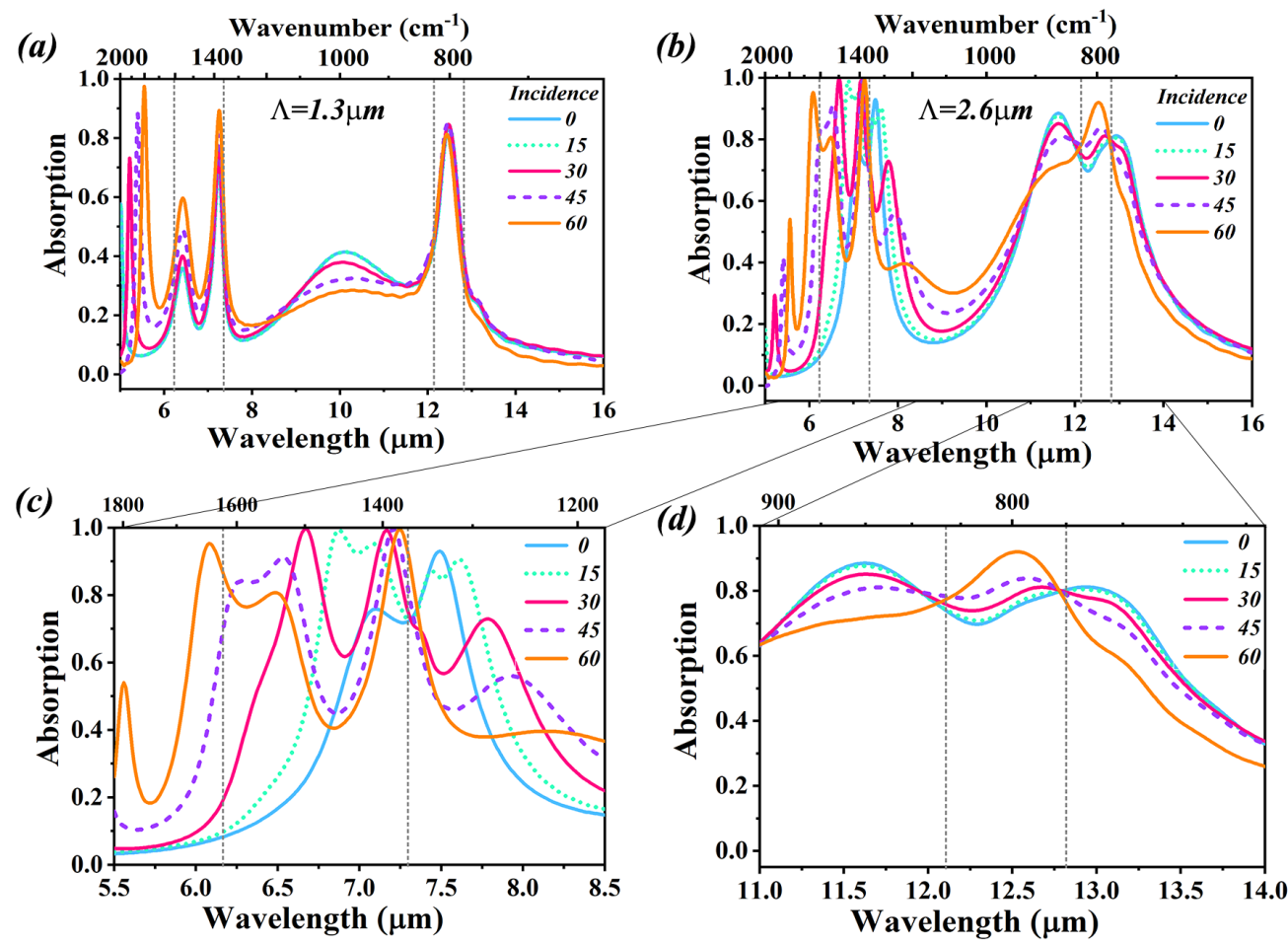
Figure S10 Devices sensitivity to injection angles which increase from 0 to 60 degrees for the sample with $t_{G e}=470 \mathrm{~nm}$, and $p=0.5$, for $\Lambda=1.3 \mu \mathrm{m}(a)$ and $\Lambda=2.6 \mu \mathrm{m}(b)$ respectively, and (c) \& (d) are area zooming of the two $R S$ bands in (b).

After isolating and understanding the response of the existing modes to the varying of the incident angle, the coupling of modes can be investigated. Figures S10b d shows similar curves as for Figure S10a except with couplings happening near both RS bands; the coupling of fundamental plasmonic lattice mode with $1-P h z$ mode near $13 \mu \mathrm{m}$ and the coupling of fundamental plasmonic cavity mode with 1-Phx and 2$P h z$ modes near $7 \mu \mathrm{m}$. For the $1-P h z$ mode and plasmonic lattice mode, as the incident angle is increased, the splitting strength decreases. This is due to the fact that at 0 incidence angle, the plasmonic lattice mode is located at $1-P h z$ resonance, and redshifts and decreases in intensity as the incidence angle is increased, hence, the coupling decreases. As the incidence angle is further increased, the plasmonic lattice mode continues to redshift and runs past the RS band, which implies coupling being tuned by simply changing the incidence angle.

Similar trend is observed for the coupling of plasmonic cavity mode with $1-P h x$ and 2-Phz modes near $7 \mu \mathrm{m}$. Near this RS band, as the incidence angle is increased, the plasmonic cavity mode redshifts as well, which is higher than that for plasmonic lattice mode due to its cavity nature. As the incidence angle is increased, the plasmonic cavity mode red shifts and couples with the 2-Phz mode, and eventually moves past the RS band. The modes below $6 \mu \mathrm{m}$ are higher order plasmonic lattice modes which redshift slightly as the incident angle increases.

It should be noted here that, for hBN thin film with less defects and good crystallinity, the absorption due to minor modes (2-Phx and 2-Phz) could be neglected. In such a device, the incidence sensitivity mainly originates from the FP cavity and the grating array and the coupling performance of optical phonons from both in-plane and out-of-plane are relatively stable for oblique incidences. The insensitivity of phonon couplings to incidence angles can be helpful for the potential application in photonic devices.

\section{References}

[1] Wang,H.; Wen, J.; Wang, W.; Xu, N.; Liu, P.; Yan,J.; Chen,H.; Deng, S. Resonance Coupling in Heterostructures Composed of Silicon Nanosphere and Monolayer WS : A Magnetic-Dipole-Mediated Energy Transfer Process; ACS Nano 2019, 13, 1739-1750.

[2] Lepeshpv,S.; Wang, M.; Krasnok, A.; Kotov, O.; Zhang, T.; Liu, H.; Jiang, T.; Korgel, B.; Terrones, M.; Zheng, Y.; Alu, A. Tunable Resonance Coupling in Single Si Nanoparticle-Monlayer WS2 Structures; ACS Appl. Mater. Interfaces 2018, 10, 16690-16697.

[3] Yoo, D.; Leon-Perez, F.; Pelton, M.; Lee, I.; Mohr, D.A.; Raschke, M.B.; Caldwell, J.D.; Martin-Morenno, L.; Oh, S.; Ultrastrong Plasmon-Phonon Coupling via Epsilon-Near-Zero Nanocavities; Nat. Photonics 2021, 15, 125-130.

[4] Todorov, Y.; Andrews, A.M.; Colombelli, R.; Liberato, S.D; Klang, P.; Strasser, G.; Sirtori, C. Ultrastrong LightMatter Coupling Regime with Polariton Dots; Phys. Rev. Lett. 2010, 105, 196402.

[5] Forn-Diaz, P.; Ultrastrong Coupling Regimes of Light-Matter Interaction; Rev. Mod. Phys. 2019, 91,025005.

[6] Huang, L.; Krasnok, A.; Alu, A.; Yu, Y.; Neshev, D.; Miroshnichenko, A.E.; Enhanced Light-Matter Interaction in Two-Dimensional Transition Metal Dichalcogenides; arXiv:2103.11064.

[7] Lepeshov, S.; Wang, M.; Krasnok, A.; Kotov, O.; Zhang,T.; Liu, H.; Jiang, T.; Korgel, B.; Terrones, M.; Zheng, Y.; Alu, A.; Tunable Resonance Coupling in Single Nanoparticle-Monolayer WS 2 Structures; ACS Appl. Mater. Interfaces 2018, 10, 16690-16697.

[8] Kodigala, A.; Lepetit, T.; Gu. Q.; Bahari, B.; Fainman, Y.; Kante, B.; Lasing Action from Photonic Bound States in Continuum; Nature 2017, 541, 196-199.

[9] Sang, T.; Dereshgi, S.A.; Hadibrata,W.; Tanriover, I.; Aydin, K.; Highly Efficient Light Absorption of Monolayer Graphene by Quasi-Bound State in the Continuum, Nanomaterials 2021, 11, 484. 